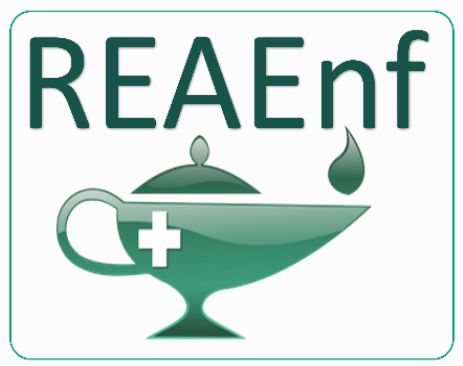

Revista Eletrônica Acervo Enfermagem
REVISÃO BIBLIOGRÁFICA

Recebido em: 5/2020

Aceito em: 7/2020

Publicado em: 9/2020

\title{
Assistência do enfermeiro frente ao paciente com hanseníase: revisão narrativa
}

\author{
Nurses' assistance to leprosy patients: narrative review
}

\author{
Asistencia de enfermeras a pacientes con lepra: revisión narrative
}

Rute Nascimento Pimentel Mendes ${ }^{1 *}$, Miréia Santana Araújo Lisboa ${ }^{1}$, Josení Ferreira Conceição Sena ${ }^{1}$, Emanuelly Moreira Miranda ${ }^{1}$, Thaís de Jesus Santos ${ }^{1}$, Nayara Tharcylla dos Santos Silva ${ }^{1}$, Robson Batista Santos².

Resumo: Este artigo buscou discutir a hanseníase, suas manifestações e seus os tipos a partir da gravidade a qual se encontra, com o objetivo de avaliar o nível de conhecimento da população quanto à hanseníase, os seus estágios e suas formas de manifestações, buscando compreender os sentimentos e o grau de conhecimento e cuidados do paciente e dos familiares em relação à doença, no período de 2008 a 2019. Trata-se de um estudo de narrativa/crítica: de caráter descritivo-discursivo, realizado através de revisão bibliográfica, coletados da base de dados SCIELO e PUBMED, foram utilizados textos completos e artigos científicos. Diante do exposto foram observados que ainda há um déficit no diálogo e nas informações por parte de alguns profissionais com os pacientes e familiares em relação aos sentimentos, anseios e receios sobre as formas e os sinais da doença. Concluiu-se que, há pouco conhecimento da população sobre a hanseníase, principalmente com relação às diversas formas de manifestações e de contágio bem como sobre tratamento e cura. Pois são de fundamental importância envolver todos nessa coleta de dados e também orientá-los sobre a doença e seus riscos, além de conscientizar a comunidade através de ações, palestras, busca ativa, e realizar os devidos encaminhamentos.

Palavras-chave: Hanseníase, Paciente, Enfermagem.

\begin{abstract}
This article sought to discuss leprosy, its manifestations and its types based on the severity it is in, with the objective of assessing the population's level of knowledge about leprosy, its stages and its forms of manifestations, seeking to understand the feelings and the degree of knowledge and care of the patient and family in relation to the disease, in the period from 2008 to 2019. This is a narrative / critical study: of a descriptive-discursive character, through bibliographic review, collected from the database. SCIELO, PUBMED data, full texts and scientific articles were used. In view of the above, it was observed that there is still a deficit in the dialogue and in the information on the part of some professionals with patients and family members in relation to the feelings, anxieties and fears about the forms and signs of the disease. It was concluded that there is little knowledge of the population about leprosy, mainly in relation to the various
\end{abstract}

\footnotetext{
1 União Metropolitana Unime Feira de Santana LTDA. Feira de Santana - BA.

*E-mail: rutenpmendes@gmail.com

2 Instituto Mantenedor de Ensino Superior da Bahia (IMES). Feira de Santana - BA.
} 
forms of manifestations and contagion, as well as about treatment and cure. Because it is of fundamental importance to involve everyone in this data collection and also to guide them about the disease and its risks, in addition to raising awareness in the community through actions, lectures, and active search, and making the necessary referrals.

Keywords: Hansen's disease, Patient, Nursing.

Resumen: Este artículo buscaba discutir la lepra, sus manifestaciones y sus tipos en función de la gravedad en la que se encuentra, con el objetivo de evaluar el nivel de conocimiento de la población sobre la lepra, sus etapas y sus formas de manifestaciones, buscando comprender los sentimientos. y el grado de conocimiento y cuidado del paciente y la familia en relación con la enfermedad, en el período de 2008 a 2019. Es un estudio narrativo / crítico: de carácter descriptivo-discursivo, a través de la revisión bibliográfica, recopilada de la base de datos. Se utilizaron datos SCIELO, PUBMED, textos completos y artículos científicos. En vista de lo anterior, se observó que todavía hay un déficit en el diálogo y en la información por parte de algunos profesionales con pacientes y familiares con respecto a los sentimientos, ansiedades y miedos sobre las formas y signos de la enfermedad. Se concluyó que hay poco conocimiento de la población sobre la lepra, principalmente en relación con las diversas formas de manifestaciones y contagio, así como sobre el tratamiento y la cura. Porque es de fundamental importancia involucrar a todos en esta recopilación de datos y también guiarlos sobre la enfermedad y sus riesgos, además de crear conciencia en la comunidad a través de acciones, conferencias y búsqueda activa, y hacer las referencias apropiadas.

Palabras clave: Lepra, Paciente, Enfermería.

\section{INTRODUÇÃO}

A hanseníase é uma patologia provocada pelo Mycobatérium leprae, uma bactéria intracelular obrigatória com tropismo preferencial nos nervos da área periférica e na camada da pele, causando alterações dos pontos sensíveis das regiões acometidas pela bactéria (RODRIGES FF, et al., 2015). É uma patologia de evolução lenta infectocontagiosa que apresenta sinais e sintomas de diversos tipos, e como manifestações principais têm-se as lesões dermatológicas e neurológicas como na pele, nos olhos, nas mãos, pés e pontos nervosos periféricos (FREITAS IASL, et al., 2008).

Há tempos a hanseníase já causava medo devido às deformidades físicas desencadeadas pela patologia, o doente não tratado, sofria preconceitos, pois para muitos a doença estava relacionada a castigo, imundícia e culpa (JUNIOR FJGS, et al., 2008). Embora a hanseníase seja infectocontagiosa tem cura, a bactéria que acomete a doença é conhecida como bacilo de Hansen, lepra, morfeia e mal- do sangue (LUNA IT, et al., 2010).

A hanseníase continua sendo um problema sério de Saúde Pública no Brasil, ainda está em segundo lugar entre os países no número de causas em todo o mundo (FREITAS IASL, et al., 2008). É caracterizada pela capacidade de ocasionar ferimento que causam deformidades com simplicidade para disseminar infecção, logo que sua evolução é crônica (JUNIOR FJGS, et al., 2008).

Entende-se que a forma principal de infecção humana ainda é na convivência domiciliar com a pessoa infectada. A principal via de contágio é respiratória, através das vias aéreas superiores e em contato próximo com o paciente bacilífero, sendo a principal porta de entrada no corpo (JUNIOR FJGS, et al., 2008).

A doença tem um período de incubação extenso, que pode vir a durar de 3 a 5 anos, devido a sua multiplicação ser lenta atingindo principalmente a pele, os nervos e os músculos. Configurando como um dos maiores problemas de saúde pública, levando a deformidade física e a incapacidade nos casos de pacientes já tratos (LUNA IT, et al., 2010).

Portanto, este estudo objetivou avaliar o nível de conhecimento da população quanto à hanseníase, os seus estágios e suas formas de manifestações, buscando compreender os sentimentos e o grau de 
conhecimento e cuidado dos pacientes e de seus familiares relacionados à doença. Além de contribuir e proporcionar tanto para o meio acadêmico quanto para os profissionais de enfermagem, maiores conhecimentos relacionados à temática abordada. Com o intuito de proporcionar melhorias no acolhimento voltado para um olhar holístico e uma assistência humanizada aos portadores da Doença de Hansen (DH).

\section{REVISÃO BIBLIOGRAFICA}

Em alguns casos a hanseníase é manifestada através de manchas avermelhadas ou esbranquiçadas em pontos específicos da pele, podendo apresentar perda de sensibilidade no local e sensação de formigamento (LUNA IT, et al., 2010). As deformidades no corpo podem ser evitadas ou minimizadas se os pacientes infectados forem diagnosticados e adequadamente tratados o mais breve possível pelo serviço de saúde. (RODRIGUES FF, et al., 2015).

A hanseníase é um filante que age dentro da célula, sendo a exclusiva classe de microbactéria que corrompe os nervos da periferia, exatamente as células de Schuann. A exclusiva via de supressão da bactéria pelo enfermo e a mais possível via de incursão deste corpo são as vias superiores (nariz e boca) por meio de contato físico por longo período, ocorre com regularidade no âmbito familiar. Logo, o convívio familiar é considerado como grande ambiente de alienação da enfermidade. A patologia não é transmitida por herenditariedade e também não há relatos de cadência durante o ato sexual (BRASIL, 2008).

A hanseníase é considerada uma patologia crônica caracterizada como uma problemática de ordem pública, passando a ser um desafio para a equipe multidisciplinar e governantes devido ao número de pessoas infectadas e pelo impacto negativo que vem causando na saúde da comunidade (RODRIGUES FF, et al., 2015).

O bacilo de Hansen é caracterizado como uma doença de infecção contagiosa e crônica provocada pelo Mycobacterium léprae, é caracterizado por sua progressão lenta, mas com alto grau de infecção e baixa patogenicidade, apresenta-se inicialmente, por meio dos sinais e sintomas dermatológicos e neurológicos, alternando entre duas formas estáveis conhecidas por tuberculóide e virchowiano e também com as formas intermediárias instáveis (JUNIOR FJGS, et al., 2008).

Hoje em dia, a doença ainda significa um potencial agravante para o Sistema de Saúde do país e em nações não desenvolvidas. A partir dos complicadores inerentes a alguma enfermidade de ascendência social e econômica, evidenciou-se que os impactos emocionais provocados pelas inabilidades físicas, provenientes da enfermidade. Estas estabelecem, na verdade, o grande motivo de estigmatização e exclusão do paciente no meio social (FREITAS IASL, et al., 2008).

A melhor forma de tratamento é través da junção dos portadores da doença em dois grupos classificando como: paucibacilares e multibacilares, pois é devido a essa divisão e classificação que se planeja o tipo de tratamento com a poli quimioterapia, definida pela OMS-Organização Mundial da Saúde (JUNIOR FJGS, et al., 2008).

Um ser humano caracterizado como um modelo imposto pela sociedade, que tem o significado de obrigar a uma condição que, de certa forma, o limita a uma classe abaixo do padrão da espécie, resultaria a ele duas condições: ou se adaptaria a viver isolado da sociedade ou se omitiria a sua real condição (JUNIOR FJGS, et al., 2008).

Para que haja um ambiente seguro para a promoção e recuperação desses portadores é necessário à educação em saúde, que englobe principalmente o uso correto do medicamento para a cura da patologia, e com isso, tornando-se também um ambiente seguro para toda família, onde todos os membros se envolvam no processo de tratamento contribuindo para melhores condições de higiene, e cuidados no processo da cura do cliente (LUNA IT, et al., 2010).

Observou-se que durante todo o processo de diagnóstico e tratamento as pessoas acometidas pela doença eram pouco abordadas sobre questões relacionadas ao convívio no âmbito familiar e no meio social (FREITAS IASL, et al., 2008). 
São questões pouco abordadas os sentimentos do doente, preocupando-se somente com o uso das medicações de forma correta, cuidados com a evolução para a incapacidade, mas não são questionados ou debatidos assuntos sobre o relacionamento no âmbito familiar e referente labor, essas questões na maioria das vezes não são abordadas durante a entrevista de enfermagem, contudo deveriam ser tão importantes quanto todo o contexto da doença (FREITAS IASL, et al., 2008).

A inclusão dos projetos de administração da patologia no Sistema de Saúde é conhecida na atualidade como o maior método para suprimir a hanseníase, para a investigação antecipada e mudanças na característica da assistência aos acometidos pela doença, favorecendo a introdução à terapêutica, a precaução de inabilidades, e a redução da mácula e do afastamento do convívio social (RODRIGUES FF, et al., 2015).

A hanseníase é considerada uma doença grave de muita relevância para os órgãos competentes, conforme o grande poder de infecção atinge principalmente a classe socioeconômica mais baixa (BRASIL, 2008). É de grande importância salientar que, durante o desenvolvimento da doença, uma grande quantidade de clientes evolui com complicações como inflamações agudas, também chamadas de reações hanseniacas. Suas manifestações também são classificadas por tipo 1 e 2 e pela resposta segundo a imunidade do hospedeiro (QUEIROZ TA, et al., 2015).

Os danos podem ser minimizados por meio de políticas públicas com o objetivo de controlar ou erradicar a doença. Logo, a Estratégia de Saúde da Família (ESF) pode colaborar como uma das principais cumpridoras por executar medidas de prevenção e tratamento, sendo que a ESF realiza 0 acompanhamento com a equipe de saúde da comunidade (JUNIOR FJGS et al., 2008).

A ação do tipo 1 é definida com a manifestação de novas irregularidades na pele, abaixo da aparência da macula, ocasionando mudanças de coloração e inchaço nas irregularidades já presentes, com exceção das dores e espessamento dos nervos (QUEIROZ TA, et al., 2015).

O tipo 2, apresentado com mais frequência é o eritema nodoso hansênico, tem como consideráveis formas clínicas o aparecimento de nódulos subcutâneos vermelhos e dilacerante, febre, dores nas juntas e mal-estar generalizado (QUEIROZ TA, et al., 2015).

As manifestações da doença têm a probabilidade de ter uma enorme desordem nos acometidos e são de relevância na justificação da inabilidade do biótipo e imperfeição definitiva. Condizem a acometimentos de infecções intensas que são definidas pelo desequilíbrio e acentuação do resultado imune a hanseníase, entretanto, até a ocasião, não se encontram marcadores laboratoriais prognosticados desses acontecimentos (QUEIROZ TA, et al., 2015).

A doença é considerada infecciosa pelo fato de a bactéria penetrar a célula nervosa com seu poder neurogênico. É uma infecção que atinge a pele e os nervos periféricos, contudo pode acometer também as articulações, mucosas oculares, saco escrotal, gânglios, entre outros órgãos, podendo ser também conhecido como doença sistêmica (BRASIL, 2008).

Depois do diagnóstico da hanseníase, a aceitação da realização ou não medicamentosa, é necessário para a transformação no modo de vida, com admissão de práticas benéficas, sendo parte constituinte do propósito da atenção, diversas vezes não entendido pelo cliente (LUNA IT, et al., 2010).

A patologia é caracterizada como uma das doenças mais antigas que age no corpo do ser humano. Há 600 anos a.C. a doença era vista como causa de punição, pecado e impureza, devido aos valores estabelecidos nos quais as pessoas acreditavam, e da forma em que se comportavam, com relação as suas praticas de acordo com o modo de vida transmitido por sua região, que moldava a forma de pensar, de agir do que fazer e de como fazer do grupo de acordo com a sua cultura (JUNIOR FJGS, et al., 2008).

É papel do profissional de enfermagem da Estratégia da Saúde da Família- ESF ofertar programas educacionais de forma continuada a toda a equipe de profissionais da unidade, assim como realizar as consultas de enfermagem que norteiam sobre a importância de rastrear os fatores de riscos com o intuído da adesão ao tratamento da doença (JUNIOR FJGS et al., 2008). 
A assistência de enfermagem é de fundamental importância nas ações para o controle da doença como forma de prevenção, de diagnóstico, de tratamento, do acompanhamento adequado dos enfermos e da realização de busca ativa. Assim como o tratamento dos clientes já incapacitados, prevenção de agravos e o gerenciamento para controle e registro da Vigilância Epidemiológica (FREITAS IASL, et al., 2008).

A doença é manifestada de diversas formas, tendo em vista que existem maneiras distintas de predominância entre regiões, o que evidência a transmissão da hanseníase (RODRIGUES FF, et al., 2015). Devido a sua característica multiformas, nem todas as pessoas estão aptas a reconhecer ou identificar seus sinais, levando assim a um diagnóstico tardio e maiores consequências.

O atendimento do (a) enfermeiro (a) é essencial e de grande relevância na assistência da comunidade e integra ao fazer parte de uma técnica coletiva de atividades dentro da unidade da família na inspeção da doença nos indivíduos infectados nos seus familiares (RODRIGUES FF, et al., 2015). O enfermeiro pertence a um sistema comum de ocupação, que empreende de forma objetiva nas intervenções de manejo da doença (FREITAS IASL, et al., 2008). Na verdade, vem envolver toda a equipe multidisciplinar, sendo que, vale destacar a importância dos Agentes Comunitários de Saúde (ACS) no processo de monitoramento e na realização da busca ativa dos casos.

A consulta com o (a) enfermeiro (a) é uma atividade ofertada pelo profissional capacitado ao cliente, no qual são observados problemas de saúde entre outras patologias, são criadas e desenvolvidas ações e atividades com o foco na promoção, proteção, recuperação e/ou reabilitação do enfermo (RODRIGUES FF, et al., 2015).

Conforme traz Brasil (2008) é relevante salientar que o enfermeiro no ato da consulta deverá "identificar os sinais e sintomas, avaliar, orientar e registrar o grau de incapacidade, realizar exame dermatoneurologico", dentre as demais atividades de acompanhamento e monitoramento do paciente, da família e da coletividade.

Fazendo com que beneficie a saúde do cliente, favorecendo ao mesmo, para aderir o tratamento, melhorando a estabilidade do cliente, reduzindo os gastos com a assistência, facilitando assim o diagnóstico de cada caso, permitindo uma assistência com resultados e qualidades, voltados às mobilizações ofertadas pelos profissionais de saúde (RODRIGUES FF, et al., 2015).

É importante contar com a colaboração da equipe de saúde multidisciplinar, pois cada um dos papeis é primordial na identificação e no cuidado com o paciente acometido com membros residentes da mesma casa e de toda área. É no primeiro contato do cliente na unidade de saúde que o enfermeiro (a) vai acolher iniciando o dialogo para conquistar a confiança e o respeito, para a partir dai poder orientar e estimular quanto a necessidade do tratamento e da e executar a notificação compulsória (BRASIL, 2008).

É dever da enfermagem acompanhar o cliente portador da hanseníase desde o diagnóstico até a alta, com um cuidado individualizado e sistematizado com a possibilidade de comunicação com o paciente, adesão ao tratamento e auxilio do autocuidado, reduzindo assim possíveis incapacidades decorrentes da hanseníase (RODRIGUES FF, et al., 2015).

No Brasil, os cuidados medicamentosos, foram iniciados por volta de 1950, trazendo expectativas para as pessoas infectadas pela lepra, no que se refere principalmente ao convívio familiar e social, pois os acometidos eram vistos como uma ameaça para a sociedade. Com isso, a terapia propiciou a reinserção desses indivíduos no meio social (ARAÚJO RMS, et al., 2017).

Embora a doença de Hansen seja de desenvolvimento lento, ela acomete inicialmente a epiderme, os nervos periféricos, a cavidade nasal, ocular, entre outras partes do corpo. Depois de um prolongado avanço, ocorrem modificações sensitivas, surgimento de vários tipos de lesões na pele, deformidades e amputações, diante disso surgem os preconceitos e consequentemente o afastamento social (VIEIRA S, et al., 2012).

Com relação ao aspecto clínico, o infectado apresenta uma elevada resistência em algumas lesões, nas quais o bacilo de Hansen é pouco evidenciado com relação às feridas, outros são bem delimitados, ou seja,

REAEnf/EJNC | Vol. 4 | e3787 | DOI: https://doi.org/10.25248/REAenf.e3787.2020 Página 5 de 7 
em menor quantidade. No aspecto de baixa potência, o bacilo é naturalmente visto, apresentando maior número de ferimento. Logo, a classificação é realizada da melhor forma possível para dar início ao plano medicamentoso. Para remanescentes ativos, o recomendado é o método paucibacilar para clientes com até cinco feridas, e acima de cinco, o multibacilar (SCHETTINI APM, et al., 2008).

A Doença de Hansen (DH) ainda é negligenciada mesmo diante de avanços terapêuticos para contê-la. Sua evolução é crônica e causa comprometimento físico, principalmente em locais menos favorecidos, onde o diagnóstico é mais demorado, podendo desencadear outras enfermidades e complicar ainda mais o estado clínico do paciente (FERREIRA AF, et al., 2019).

Mesmo com o empenho para extinguir a hanseníase, o número de pessoas acometidas que evolui com a hansen é muito considerável em todo o mundo (GAMA ES, et al., 2013). A morte pela lepra ainda é tratada com descaso por alguns, principalmente quando se trata sobre investigação e atividade de vigilância e controle (FERREIRA AF, et al., 2019).

De acordo com Brasil (2017) "A hanseníase é transmitida por meio do contato próximo e prolongada de uma pessoa suscetível à doença com um portador da hanseníase e que não esteja sendo tratado". Isso se dá, porque a forma de contágio é inevitável para quem desconhece a doença e seus sintomas. O contágio da doença é através de gotículas salivares, espirros e uso de objetos contaminados, deixando assim os familiares em estado de vulnerabilidade, mas acredita-se que tem pessoas que possuem uma defesa natural contra o bacilo (BRASIL, 2017).

Cliente identificado com malformação de grau 2 representa pequeno grau de entendimento a respeito da doença em coletividade, exceto a habilidade limitada da função de detectar a doença prematuramente (DEPS PD, FLORIANO MC, 2017). A doença quando acomete um adolescente no meio em que vive a probabilidade de transmissão ativa é maior se não houver rastreamento (DEPS DP, FLORIANE MC, 2017).

A hanseníase dispõe de um autopoder de desenvolvimento e propagação. Para isso se faz necessário ter conhecimento da região com alto risco de infestação para que se possa conter a disseminação da doença. Nesse cenário é imprescindível que sejam traçados planos e metas que cumpram as singularidades de cada localidade (BRASIL, 2014).

Diante do que foi exposto, é importante ressaltar que o indivíduo acometido pela patologia e que esteja seguindo uma terapêutica correta poderá conviver naturalmente com seus familiares, colegas de atividade laboral e amigos. Sendo que, é fundamental que não só os enfermeiros, mas que todos estejam orientados, cientes e em alerta para as formas da doença e seus sinais e sintomas (RODINE FCB, 2010).

\section{CONSIDERAÇÕES FINAIS}

Considerando o que foi exposto, observou-se déficit na capacitação de profissionais para atendimento ao portador da hanseníase, assim como, a necessidade de acompanhar e instruir os familiares e/ou cuidadores quanto aos cuidados relacionados à higiene, ao manuseio de objetos pessoais, administração de medicação e quanto a saúde psicológica desse individuo no ambiente domiciliar. Logo, pode-se constatar que seria necessário que os profissionais buscassem conhecer melhor a rotina desse cliente para a partir dessas informações traçar estratégias para conhecer e entender os sentimentos desses pacientes frente à doença, diante da família e da sociedade. E as realizações de mais ações e palestras sobre os tipos e formas da hanseníase e suas manifestações, pois, os casos de infecções são maiores no âmbito familiar por desconhecimento das formas de contágio e cuidados com o portador.

\section{REFERÊNCIAS}

1. ARAÚJO RMS, et al. Análise do perfil Epidemiológico da Hanseníase. Rev enferm UFPE on line. Recife. Pernambuco, 2017: 11(9): 3632-41.

2. BRASIL, 2008. Ministério da Saúde. Secretaria de Atenção à Saúde Departamento de Atenção Básica. Vigilância em Saúde Dengue, Esquistossomose, Hanseníase, Malária, Tracoma e Tuberculose. Brasília, 2008. 
3. BRASIL, 2014. Ministério da Saúde. Universidade de Brasília. Hanseníase Avanços e Desafios. Brasília, 2014.

4. BRASIL, 2017. Ministério da Saúde. Secretaria de Vigilância em Saúde. Hanseníase. Vitória, 2017.

5. DEPS PD, FLORIANO MC. Hanseníase. 1e ed. Vitória: E. Santo, 2017; 180p.

6. FREITAS CASL, et al. Consulta de enfermagem ao portador de hanseníase no território da Estratégia da Saúde da Família: percepções de enfermeiro e pacientes. Brasília. D. Federal, 2008; 61: 757-63.

7. FERREIRA AF, et al. Mortalidade por hanseníase em contextos de alta endemicidade: análise espaço-temporal integrada no Brasil. Brasil. Bahia, 2019; 43: e 87.

8. GAMA RS, et al. Um novo método de análise molecular e sorológica integrado para prever novos casos de hanseníase entre contatos domiciliares. Suíça. Berna, 2019; 13 (6): e 0007400.

9. JUNIOR FJGS, et al. Assistência de enfermagem ao portador de Hanseníase: abordagem transcultural. Brasília. D. Federal, 2008; 61: 713-7.

10. LUNA IT, et al. Adesão ao tratamento da Hanseníase: dificuldades inerentes aos portadores. Brasília. D. Federal, 2010; 63(6): 983-90.

11. QUEIROZ TA, et al. Perfil clínico e epidemiológico de pacientes em reação hansênica. Rio Grande do Norte. Natal, 2015; 36: 185-91.

12. RODINI FCB, et al. Prevenção de incapacidade na hanseníase com apoio em um manual de autocuidado para pacientes. Fisioterapia e Pesquisa, São Paulo. S. Paulo, 2010; 17(2): 157-66.

13. RODRIGUES FF, et al. Conhecimento e prática dos enfermeiros sobre hanseníase: ações de controle e eliminação. Brasília. D. Federal, 2015; 68(2):297-304.

14. SCHETTINI APM, et al. Hanseníase históide de localização restrita. Manaus. Amazonas, 2008; 83(5):470-2.

15. VIEIRA S, et al. Métodos de avaliação e tratamento da hanseníase: uma abordagem fisioterapêutica. São Paulo. S. Paulo, 2012; 11(1):179-184. 